\title{
RESULTADOS EN LAS LENGUAS ROMANCES DE ADJETIVOS CON DESINENCIAS DERIVADAS DE -UTTUM
}

\author{
RICHARD LAURENT \\ Universidad de California, Berkeley
}

\section{INTRODUCCIÓN}

En los últimos años, Harris-Northall ${ }^{1}$ y Malkiel $^{2}$ han demostrado que los participios pasados con desinencias derivadas de -ŪTUM, etc. se utilizaban todavía en la alta Edad Media en España. Williams ${ }^{3}$ ha afirmado lo mismo en cuanto a los participios pasados en -udo del portugués. Harris-Northall ${ }^{4}$ señala que algunos de los participios pasados que aparecen en textos españoles medievales son de verbos no atestiguados más al norte de los Pirineos, como comudo 'comido', seudo 'sido'. Otros participios pasados en -udo que se encuentran en el español medieval parecen ser más innovadores que las formas galas de la misma época, por ejemplo, ardudo 'ardido', prometudo 'prometido', y traudo 'traído' en español medieval, versus fr./occit. ars $<\bar{A} R S U$, fr. promis y occit. promes $<$ PRŌMISSU, fr./occit. trait $<$ TRACTU. Por otra parte, formas como atrevudo 'tributo' y batudo 'batido' del español antiguo pueden representar una continuación de ATTRIBŪTU 'asignado' y BATTŪTU, lo cual sugiere que este tipo de derivación mantenía en esta época su popularidad.

Otra pregunta que surge en relación con las observaciones ya hechas con respeto a los participios pasados en -udo en el español antiguo es la si-

\footnotetext{
${ }^{1}$ Ray Harris-Northall, «The Old Spanish Participle in -uDo: Its Origin, Use, and Loss», Hispanic Review, 64, 1996, págs. 31-56.

2 Yakov Malkiel, «La pérdida del participio en -uDo», Nueva Revista de Filología Hispánica, XL.1, 1992, págs. 11-28.

${ }^{3}$ Edwin B. Williams, From Latin to Portuguese: Historical Phonology and Morphology of the Portuguese Language, 2.a ed., Philadelphia, University of Pennsylvania Press, 1962, págs. 185, 237-238, 241-242.

${ }^{4}$ Harris-Northall, op. cit., págs. 36-37.
} 
guiente: ¿cómo percibiría un hablante del romance ibérico centrooccidental un participio pasado en -ŨTU pronunciado por un recitador de obras líricas o épicas de una zona al norte de los Pirineos? Al escuchar una obra recitada en francés antiguo o en occitano antiguo (sin tomar en cuenta las diferencias de caso), el hablante del romance ibérico oiría las terminaciones [yt] para formas masculinas singulares, e [yðe] para formas femeninas singulares. Hay que preguntarse si un español o un portugués de la época podría sacar sentido a estas formas ya que, por una parte, ni [y] ni [ə] eran fonemas del romance ibérico centrooccidental, y por otra parte, existían restricciones fonotácticas que no permitían que las palabras terminasen en [t].

Aunque unos cuantos participios pasados en -udo que se encuentran en el español antiguo seguían usándose como adjetivos en una época temprana del desarrollo de la lengua moderna, por ejemplo tenudo 'obligado por gratitud' y sabudo 'sabio', hoy los participios pasados de tener y saber sólo pueden ser tenido y sabido. Actualmente las dos variantes adjetivales en -udo están en desuso ${ }^{5}$. Otros derivados verbales también han cambiado de forma con el paso del tiempo: entendudo 'entendido, experto' en español antiguo se ha convertido en la forma de la $4 .^{a}$ conjugación, entendido ${ }^{6}$.

Dado que parece que se ha solucionado la cuestión de los participios pasados españoles derivados de -ŪTUM, es el momento oportuno de examinar los adjetivos con desinencias parecidas en toda la Romania. A partir de las bases establecidas por Malkiel, esta investigación presenta un análisis panromance del éxito o la falta de éxito de varios tipos de desinencias adjetivales que hacen referencia a las partes del cuerpo y a las emociones.

\section{LA FUNCIÓN DE -ŨTUM EN LA MORFOLOGía DEL LATÍN}

Los hablantes de latín clásico (LC) utilizaban la desinencia -ŨTUM (más tarde -ŪTU) para formar tanto los participios pasados de bases verbales con $-u$ como las formas adjetivales de bases nominales en $-u$. Las dos formas eran poco comunes ${ }^{7}$. A parte de una veintena de participios pasados como

\footnotetext{
${ }^{5}$ En contraste, en italiano se mantiene el p.p. saputo 'sabido' y un adjetivo diminutivo derivado del p.p. saputello 'sabelotodo'.

${ }^{6}$ Thomas Montgomery, «A Latin Linguistic Icon Readapted in Proto-Romance and in Medieval Spanish», Hispanic Review, 1995, 63, pág. 150. Nótese, sin embargo, la forma inteligentudo en el español mexicano no-estándar, que pertenece aproximadamente al mismo campo semántico, y en la que el sufijo mantiene su productividad.

${ }^{7} \mathrm{La}$ función de los dos tipos a veces era la misma: los antiguos participios pasados como ACŪTU 'agudo' y MINŪTU 'menudo' ya eran principalmente adjetivales en la época del LC. Seguían existiendo en romance solamente con el significado que tienen hoy en español: agudo, menudo.
} 
SECŪTU 'seguido', SOLŪTU 'suelto', vOLŪTU 'vuelto', en LC había unos adjetivos derivados de sustantivos de la $4 .^{\text {a }}$ conjugación como ASTŪTU 'astuto' de ASTU 'astucia' o VERŪTU 'armado con dardo o jabalina', de VERU 'dardo, jabalina'.

Algunos adjetivos en -ŪTU se usaban, a veces con significado aumentativo, para referirse a partes del cuerpo humano o de un animal. Muchos eran derivados de sustantivos en $-u$ de la $4 .^{a}$ declinación, por ejemplo CORNŪTU 'cornudo' de CORNU 'cuerno', y MANŪTU 'manota' de MANUS 'mano'. En LC la desinencia -ŪTU se utilizaba también con adjetivos y sustantivos de otras clases, por ejemplo: CĀNŪTU 'cano' de CĀNA/CĀNUS/CĀNU 'blanco grisáceo' de la 1. a/2. a declinación y NĀSŪTU 'narigudo' de NĀSUS 'nariz' de la $2 .^{a}$ declinación (cf. nasudo, raro en español) ${ }^{8}$.

\section{LA SUERTE DE CUATRO SUFIJOS ADJETIVALES QUE HACEN REFERENCIA A PARTES DEL CUERPO}

En latín clásico se utilizaban varios sufijos además de -ŪTUM para formar adjetivos que hacían referencia a partes del cuerpo humano o de animales, a saber, -ĀTUM, -ITTUM, -EUM y -ŌSUM. Los primeros dos se utilizaban también para formar los participios pasados de verbos regulares de las conjugaciones $1 .^{\mathrm{a}}$ y $4 .^{\mathrm{a}}$ respectivamente; los dos últimos solamente tenían función adjetival.

De los sufijos del LC que hacían referencia a las partes del cuerpo, -ĀTUM casi siempre denotaba posesión de alguna parte del cuerpo, sin referencia al tamaño de dicha parte y sin ninguna connotación emotiva. Esto parece ser verdad en el caso de BARBĀTUM 'barbado', CRISTĀTUM 'crestado', DENTĀTUM 'dentado', MENTULĀTUM 'que tiene pene', OCULĀTUM 'que tiene ojos', PENNĀTUM o PINNĀTUM 'que tiene plumas', PEDĀTUM 'de pie', ROSTRĀTUM 'que tiene pico'. Sin embargo, CERVICĀTUM 'cabeza dura, obstinado', CORDĀTUM 'prudente, sabio' y LINGUĀTUM 'elocuente' sí tenían connotación emotiva y no significaban simplemente 'que tiene cuello', 'que tiene corazón' y 'que tiene lengua'. Por otra parte MAMMEĀTUM 'pechugón' y CAPILLĀTUM 'que tiene pelo' y también 'de pelo largo', podían tener además un valor aumentativo.

\footnotetext{
${ }^{8}$ Los participios pasados tienen su origen en los adjetivos verbales. Véase Manu Leumann, Lateinische Laut- und Formenlehre, $2 .^{\mathrm{a}}$ ed. (Handbuch der Altertumswissenschaft, vol. 2:2:1), Munich, C. Beck, 1977, págs. 593-598 para una discusión de los orígenes del sistema de las formas perfectas de los verbos en [w] o [u] en LC. Para una discusión de las bases nominales en $-u$ véase Carl Darling Buck, Comparative Grammar of Greek and Latin, Chicago, University of Chicago Press, 1933, págs. 198-201.
} 
Algunos adjetivos que hacen referencia a partes del cuerpo terminaban en -ITTUM, por ejemplo AURITTUM 'de orejas largas' o CRĪNITUM 'de pelo largo'. Otros terminaban en -EUM, por ejemplo CORPOREUM 'carnoso', PULMONEUM 'del pulmón', OCULEUM 'de vista aguda; perspicaz' o OSSEUM 'huesudo'. La mayoría parece tener valor aumentativo. Sin embargo, con el paso del tiempo tanto -ITTUM como -EUM se hicieron menos productivos como desinencia para adjetivos que se refieren a partes del cuerpo.

En LC los adjetivos con la desinencia -ōSUM que hacen referencia al cuerpo por lo general tienen valor aumentativo. Algunos ejemplos son: CAPILLŌSUM 'cabelludo', CARNŌSUM 'carnoso', LABIŌSUM o LABEŌSUM 'labio grueso', MAMMŌSUM 'pechugón', NERVŌSUM 'nervudo', PECTORŌSUM 'de mucho pecho', PILŌSUM 'piloso', SÆTŌSUM 'cerdoso', VENTRIŌSUM 'ventroso' y VILLŌSUM 'velludo'. Esta terminación se utilizaba también en términos médicos, por ejemplo, JECINERŌSUM o JECORŌSUM 'enfermo del hígado', VENŌSUM 'venoso' o VESICULŌSUM 'vesiculoso'. La desinencia -ŌSUM se utilizaba también para formar adjetivos referidos a las emociones o al carácter, por ejemplo, CERVICŌSUM 'cabezón', FRONTŌSUM 'sinvergüenza', GULŌSUM 'goloso', LINGUŌSUM 'hablador' y RABIŌSUM 'rabioso'.

Los puristas desaprobaban el uso de esta desinencia, tal y como se declara en el artículo n. ${ }^{\circ} 211$ del Appendix Probi: RABIDUS non RABIŌsus ${ }^{9}$. Sin embargo, formas como MEMBRōsus 'membrudo' que aparecen en las Notce Tironienses sugieren que el sufijo -ōsu mantuvo su popularidad por lo menos en el habla vulgar ${ }^{10}$.

En las lenguas que descienden del latín, por lo general las terminaciones derivadas de -ITTU y -EU con función adjetival desaparecieron, las del último grupo posiblemente por la reducción fonética (en muchas zonas el resultado fue [jo]), y las del primero, a pesar del extenso uso de los reflejos de -ITTU como terminación para los participios pasados, muchos de ellos también usados como adjetivos. Al contrario, los reflejos de -ĀTU florecieron tanto con función de adjetivo como de participio pasado. Los adjetivos que hacen referencia a partes del cuerpo y que tienen desinencias derivadas de -ōsu entraron en fuerte competencia con los adjetivos con reflejos de tipo -ŪTU en romance.

Como se ve en la sección 9, la tendencia que se nota en español hacia una preferencia por las desinencias derivadas de -ōsu sobre las derivadas de -ŪTU, es más marcada en el rumano. En la sección 8 se ve que la desinen-

\footnotetext{
${ }^{9}$ No se sabe la fecha exacta del Appendix Probi, pero se recopiló en una época en que todavía se seguían las reglas del LC en la escritura.

${ }^{10}$ Ferdinand Brunot y Charles Bruneau, Précis de grammaire historique de la langue française, 2. ${ }^{a}$ ed., París, Masson, pág. 114.
} 
cia con función adjetival (y de participio pasado) más productiva, la que deriva de $-\bar{A} T U$, sigue ganando popularidad en romance, como también ocurre en todas partes y hasta cierto punto en el caso de las terminaciones derivadas de -ōsu.

4. UNA MUESTRA DE LOS ADJETIVOS EN -UDO QUE HACEN REFERENCIA A PARTES DEL CUERPO EN ESPAÑOL Y PORTUGUÉS, Y CONSIDERACIÓN DE ADJETIVOS CATALANES EN - UT QUE TIENEN ESTE SIGNIFICADO

En español moderno hay muchos adjetivos en -udo que hacen referencia a partes del cuerpo, por ejemplo, bocudo, cejudo, dentudo, cornudo. A menudo son paralelos a los adjetivos en $-u$ del francés, como barbu 'barbudo', bossu 'jorobado', ventru 'ventrudo', poilu 'peludo', têtu 'cabezón', goulu 'glotón', y a los adjetivos italianos 'en -uto como barbuto, occhiuto, gambuto ${ }^{11}$.

Rainer hace una observación que evidentemente se puede aplicar a otras lenguas romances, en la que divide las palabras que en italiano terminan en -uto en tres clases: a) participios pasados adjetivizados como compiuto 'terminado, realizado' del infinitivo compiere, b) términos opacos como arguto 'perspicaz, ingenioso', c) palabras denominativas como barbuto 'barbudo' de $b a r b a^{12}$. El resto de esta investigación examina en cada lengua el tercer tipo de adjetivo que considera Rainer.

A dos adjetivos catalanes, panxut 'panzudo' y camut 'de piernas bien formadas', se les puede añadir un sufijo opcional -arr-: panxarrut, cama$r r u t^{13}$. Posiblemente tenga relación con éstos el cat. geperut 'jorobado', de gep 'joroba' $<\mathrm{GIBBU}$, que, aunque no tiene vibrante, demuestra una extensión silábica ausente en el préstamo español chepudo ${ }^{14}$. A veces la presencia o la ausencia de un sufijo marca una distinción semántica: la forma larga del port. linguarudo tiene el significado adicional de 'cotorrear, parlotear', a diferencia de la forma corta lingudo 'lengua larga', un simple aumentativo ${ }^{15}$.

\footnotetext{
${ }^{11}$ Malkiel, op. cit., pág. 19.

12 Franz Rainer, I nomi di qualità nell'italiano contemporaneo (Wiener romanistische Arbeiten, 16), Vienna, Braunmüller, 1989, págs. 213-214.

${ }_{13}$ Salvador Oliva y Angela Buxton, Diccionari català-anglès, Barcelona, Enciclopèdia catalana, 1986, págs. 172,608 .

${ }^{14}$ En español también existe giboso<GIBBōsu (Joan Corominas, 1973, Breve diccionario etimológico de la lengua castellana, 3. ${ }^{\text {a }}$ ed., Madrid, Gredos, 1973). Esta forma parece ser un cultismo, ya que la derivación esperada hubiera dado *geboso. En valenciano existe una variante gepelut, que contiene un infijo lateral.

${ }^{15}$ Cándido de Figueiredo, Novo dicionário da lingua portuguesa, 6. ed., Lisboa, Bertrand, 1937.
} 
El adjetivo español testarudo posiblemente contenga el antiguo infijo líquido ibero. Las últimas dos sílabas de este adjetivo a veces sufren a manos de los hablantes una metátesis que da como resultado testaduro ${ }^{16}$.

Algunos adjetivos catalanes en - $u$ t incorporan un sufijo con $[\mathrm{g}]$, sin duda con función antihiática, por ejemplo, bonyegut 'magullado, con protuberancias', de bony 'protuberancia, chichón', y las formas relacionadas puntegut y punxegut 'afilado, agudo', de punt, punxa, además de forcegut 'fuerte, duro' y ronyagut 'roñoso, sarnoso', de ronya 'sarna, picazón'. Estos adjetivos se parecen a los participios pasados, sin embargo no pertenecen a ningún verbo de la $2 .^{\mathrm{a}} / 3 .^{\mathrm{a}}$ conjugación, aunque existe punxar, de la $1 .^{\mathrm{a}}$ conjugación con el participio pasado regular punxat 'chasqueado, aguijado'.

Incrementan la riqueza de los adjetivos que en catalán hacen referencia a partes del cuerpo carabrut 'cara sucia' de carabrú 'moreno', y la forma larga llargarut 'alto y delgado' de llarg 'largo', ninguno de los cuales parece tener cognados en otras lenguas romances. A un catalán corpulento se le puede llamar sacsonut de sacsó 'pliegue de gordura', un término con sufijo ibero aparentemente relacionado con la frase adverbial $a$ betzef 'abundantemente, pródigamente', cf: it. a bizeffe 'idem'. En cuanto a las aves, el adjetivo catalán crestut 'crestudo' se ha nominalizado con el significado de 'gallo', al igual que becut 'pico largo', que se ha nominalizado con el significado de 'zarapito', un ave ribereña; este proceso semántico ha ocurrido al revés en el caso del catalán arpellut 'con garras', aparentemente de arpella 'especie de halcón'.

Por la desagradable connotación que tiene, por ejemplo en italiano cornuto 'cornudo', en portugués, en vez de cornudo se suele utilizar el adjetivo chifrudo 'con cuerno' de chifre, hasta cuando se hace referencia a animales como el toro o el ciervo. Por otra parte, entre los hablantes de portugués se encuentran dos formas nominalizadas de adjetivos que hacen referencia a partes del cuerpo, o chifrudo 'el que tiene cuernos' y o rabudo 'el que tiene rabo', las dos con referencia al diablo. En español el adjetivo cabelludo aparece solamente en la frase cuero cabelludo. Sin embargo, la existencia de un entremés del teatro popular del siglo XVI titulado «Los gigantes y cabezudos» sugiere que este adjetivo posiblemente haya tenido un uso más extendido en el pasado.

${ }^{16}$ Para más discusión de los sufijos españoles -arro, -urro, -orro, véase Ramón Menéndez Pidal y Antonio Tovar, "Los sufijos con -rr- en España y fuera de ella, especialmente en la toponimia (1)», Boletín de la Real Academia Española XXXVIII, CLIV, 1958, págs. 161-214. Véase también Gerhard Rohlfs, «Das spanische Suffix -arrón und verwandtes», Archiv für dem Studium der neuren Sprachen, CLXXXII, 1943, págs. 118-122. Cuando se usan en la Península Ibérica estos sufijos suelen tener una connotación peyorativa o aumentativa, o ambas, como se ve en el cat. gossarro 'perro grande y feo', de gos (de origen incierto), camarra 'pierna grande y fea', basado en cama $<\mathrm{GAMBA}$, esp. cacharro 'olla' pero también 'cachivache inservible', basado en cacho. 
Según Lang, los adjetivos españoles en - $u d o$ que hacen referencia a partes del cuerpo suelen ser peyorativos, pero pueden tener connotación favorable si el hablante considera una "abundancia de la base» de la que se trata como rasgo físico atractivo ${ }^{17}$. Lang contrasta lo que considera positivo (una mujer tetuda) con lo que considera negativo (un hombre panzudo).

De igual manera, Frota observa que -udo en portugués tiene una connotación implícita de exceso. Las bases nominales a las que se añade son casi siempre semánticamente neutras, y en general -udo les añade un matiz peyorativo. Sin embargo, Frota afirma que si la base se refiere a una parte del cuerpo muy apreciada como bunda 'caderas de la mujer', el tamaño grande de esa parte se valora positivamente y por lo tanto -udo tiene una connotación positiva. Otros adjetivos positivos de esta clase son, por ejemplo, el port. polpuda 'apeteciblemente rollizo, jugoso, excitante', que tiene un significado paralelo en italiano. La percepción que tiene el hablante del sufijo depende en parte de su propia experiencia ${ }^{18}$.

En términos morfológicos, el español tiene mayor preferencia por los adjetivos con desinencias derivadas de -ōsu que el catalán. En catalán existen tanto ramut como ramós, pero el español tiene solamente el adjetivo ramoso con el mismo significado. En catalán se encuentra el adjetivo canut, que se parece al it. canuto y el fr. chenu, pero en español el adjetivo con este significado es canoso (aunque en español antiguo se utilizaba encanudo). En español, según Lang, lo esperado hubiese sido que los adjetivos en -udo se formaran con -oso en vez del primer sufijo, ya que -oso normalmente denota abundancia de la base ${ }^{19}$. Como se ve en la sección 8 , el español efectivamente demuestra esta tendencia.

\section{AdJETIVOS QUE EN SU MAYORÍA HACEN REFERENCIA A LAS PARTES DEL CUERPO Y QUE TIENEN RESONANCIA EMOTIVA}

Ampliaciones en el significado de otros adjetivos romances en -ŪTU han hecho que éstos se refieran a cualidades humanas, normalmente a cualidades poco deseables. Por ejemplo, el fr. têtu 'cabezón', de tête 'cabeza' y goulu 'glotón', de gueule 'morro, garganta' son paralelos al cat. cabut 'cabezón' y golut 'glotón', de cabeça 'cabeza' y gola 'garganta'.

\footnotetext{
${ }^{17}$ Mervyn F. Lang, Spanish Word Formation: Productive Derivational Morphology in the Modern Lexis, London, Routledge, 1989, pág. 116.

${ }_{18}$ Maria Paula Frota, «A espressão do pejorativo em contruções morfológicas», On Spanish, Portuguese, and Catalan Linguistics, ed. John J. Staczek, Washington, Georgetown University Press, 1988, págs. 83-90.

19 Lang, op. cit., pág. 116.
} 
El cat. morrut 'labio grueso, malhumorado', que se basa en otra parte del cuerpo, viene de morro 'labio'. El cat. caparrut 'testarudo' aparentemente viene de caparra 'dolor de cabeza' (posiblemente de 'cabeza hinchada' en su origen) cf. el esp. caparra, de posible procedencia mozárabe. De igual manera, otra amplificación de significado se encuentra en el cat. llengut 'de lenguaje sarcástico' de llengua, que se parece al it. arcaico linguto. En cuanto al tipo de cuerpo que tiene uno, un recipiente motiva un adjetivo en catalán, boterut 'fornido, robusto', de bóta 'barril', en el que la sílaba de en medio, -er- representa un morfema vacío.

A parte de denotar simplemente tamaño, en español algunos adjetivos en - $u d o$ que hacen referencia a partes del cuerpo tienen una carga emotiva o caracterológica. El esp. patudo, de pata, quiere decir 'de pies grandes', mientras patoso tiene el significado de 'torpe'. Ceñudo, de ceño, con significado de 'malhumorado', se parece al port. carrancudo, por su semántica y su desinencia, si no por su base. Tanto el esp. pechudo (¿dudoso?) como el port. peitudo, literalmente 'pechugón', tienen el significado de 'valiente, atrevido'. Compárese este adjetivo con el español americano agalludo 'atrevido', de agalla, con significado figurativo de 'fortaleza, valor' y que también puede significar 'ambicioso' o 'avaro'.

Siguiendo con los adjetivos relacionados con el carácter, tanto el cat. queixut como el port. queixudo 'de mandíbula saliente' también tienen el significado de 'testarudo, terco', sin duda por la imagen de determinación que demuestra el prognato. Entre las lenguas que tienen este tipo de adjetivo que se refiere al cerebro, el it. arcaico cervelluto de CEREBELLU y el esp. sesudo de seso $<$ SĒNSU 'sentimiento, juicio, actitud' tienen el significado generalmente favorable de 'razonable, inteligente'. Al contrario, el port. sisudo quiere decir 'severo, ceñudo'. De hecho, CEREBRōSU del LC ya tiene el significado peyorativo de 'impetuoso, de genio vivo'.

A excepción de los términos derivados de palabras que significan 'seso', el cat. fetjut 'lento, flemático' de fetge 'hígado'<* FICATU 'con higos' es el único adjetivo en - $u t$ que se forma a base del nombre de un órgano interno. El cat. verinut 'venenoso', originalmente aplicado a las culebras, viene de veri 'veneno' $<$ VENĒNU 'droga, veneno', que (después de la disimilación) ha adquirido el significado de 'rencoroso, malicioso' cuando tiene referente humano. El cat. ceballut 'obstinado' viene de ceballot 'cebolleta; zoquete'.

En español, un adjetivo en -udo que no tiene como base un sustantivo que denote una parte del cuerpo es tozudo, de toza, que sin embargo se refiere a una característica humana (cf. en inglés blockhead 'zopenco, zoquete'). El adjetivo cortezudo, de corteza, también se basa en el nombre de una parte de una planta, y tiene el significado no sólo de 'corteza gruesa', sino también de 'bruto, descortés' cuando se refiere a una persona, sin duda 
por la aspereza de la corteza. De igual manera, conchudo, de concha, puede significar no solamente 'cubierto de conchas' sino también 'astuto, mañoso', una imagen posiblemente basada en el escondimiento que ofrece una concha ${ }^{20}$. En el español de América, conchudo tiene varios sentidos, algunos «intraducibles».

En el español actual, los adjetivos en -udo que se refieren al carácter humano también pueden tener como base sustantivos o adjetivos abstractos. Estos incluyen los adjetivos de matiz positivo concienzudo y corajudo, y los negativos pacienzudo y confianzudo. Otros adjetivos denominativos son sañudo de saña, cachazudo de cachaza y berrinchudo de berrinche ${ }^{21}$. Por otra parte, el portugués tiene dos palabras con significado de 'musculoso, fuerte': forçudo, que comparte con otras lenguas, pero también mucudo de muque 'fuerza'. Lang considera que en español este tipo de formas es atípico e improductivo, a diferencia de los adjetivos en -udo, una desinencia que «attaches preferrably to designations of parts of the body» ('se añade preferentemente a designaciones para partes del cuerpo') ${ }^{22}$. Malkiel considera que $-u d o$ como desinencia adjetival es poco estable en español: a menudo entra en competencia con desinencias como -ado, -oso y -ón 23 .

Como se demuestra en la sección 6 , la terminación $-u$ con función adjetival sufre en francés una inestabilidad parecida: con el paso del tiempo $-u$ ha sido reemplazado a menudo por -é o -eux.

Cuando se añade a sustantivos abstractos, la terminación - $u t$ en catalán se puede referir a otras cualidades humanas, por lo general a cualidades poco halagüeñas. Estos términos incluyen rabiüt 'rencoroso', de ràbia, geniüt 'mal genio', de geni 'carácter, genio', calmut 'indolente, perezoso', de calm. Otro adjetivo de este tipo es rampellut 'impulsivo, salvaje', de rampell 'capricho, explosión de ira'. Dos adjetivos catalanes en -ut vienen de verbos: tossut 'obstinado', de tossar 'topetar' y saberut 'docto, sabelotodo', de saber. La sílaba -er posiblemente sea derivada del infinitivo, a diferencia de la forma del español arcaico sabudo, pero más probablemente representa un morfema vacío ${ }^{24}$.

\footnotetext{
20 David Mighetto y Per Rosengren, Diccionario reverso (Spansk Baklängesordbok), Göteborg, Institutionen för Romanska Språk, 1985, y Diccionario de la Real Academia Española, Madrid, Espasa-Calpe, 1992. No incluimos los adjetivos en -udo/-ut en español y en catalán derivados de partes de plantas pero que no tienen ninguna carga emotiva.

${ }^{21}$ Montgomery, op. cit., pág. 150.

${ }^{22}$ Lang, op. cit., pág. 116. Sin embargo, si se toman en cuenta algunas palabras del español americano como platudo de plata y macanudo de macana, la opinión de Lang posiblemente no se pueda aceptar sin modificaciones.

${ }^{23}$ Malkiel, op. cit., pág. 18

${ }^{24}$ Oliva y Buxton, op. cit., passim.
} 
En otros tres adjetivos de este tipo - gollut 'que tiene bocio', de goll 'bocio', clotut 'lleno de hoyuelos', de clot 'hueco; hoyuelo', y granellut 'granujoso', de gra 'semilla; grano (en la cara)' - la terminación -ut sólo tiene valor adjetival, pero normalmente tiene referente humano. El adjetivo catalán traçut 'listo, hábil', de traça 'plan, intención' hasta suena favorable.

Si carajudo (esp.) fuera simplemente el aumentativo de carajo, el cognado nominal catalán carall se ha vuelto arcaico con ese significado, y actualmente se usa como interjección como en inglés hell 'caramba' o damn 'maldito sea'; entre sus derivados, el sustantivo masculino singular carallot quiere decir 'tonto, bobalicón', mientras el sustantivo femenino singular $c a$ rallada significa 'bagatela' y el sustantivo plural carallades, 'travesura, chismes' ${ }^{25}$. En este caso también existen en italiano formas semánticamente relacionadas como sg. cazzata y pl. cazzate de cazza 'pene', aunque parece que ningún adjetivo en -uto se deriva de esta raíz.

\section{El DECLIVE DE LOS ADJETIVOS FRANCESES EN $-U$}

La terminación - $u$ del francés moderno, como -uto en italiano, - $u d o$ en español y portugués y -ut en catalán y rumano puede servir simplemente para formar adjetivos denominativos que se refieren a la forma que tiene una cosa. Unos ejemplos en francés son crochu 'encorvado, torcido', de croc, fourchu 'bifurcado', de fourche 'horca' y pointu 'puntiagudo', de point 'punto'. Sin embargo, cuando se refiere a las plantas, esta desinencia parece tener cierto valor aumentativo, por ejemplo branchu 'ramoso', cossu 'que tiene muchas vainas (de semillas)', de cosse, feuillu 'frondoso', herbu 'cubierto de hierba' y mossu 'musgoso'. En una clase aparte se encuentra co$c u$ 'cornudo', de origen incierto.

En francés antiguo existían versiones de casi todos los adjetivos modernos en $-u$ y muchos más. Algunos han adquirido otras desinencias que carecen de valor aumentativo. Entre los adjetivos que se refieren a las partes del cuerpo del cuello para arriba se encuentran ant. fr. nasu 'narigudo', lav$r u$ o levru 'labio grueso', dentu 'dentudo' (mod. denté 'dentado') y oreillut 'orejón, orejudo'; en francés antiguo testu tenía el significado tanto de 'cabezota' como de 'terco, obstinado'. Haciendo referencia al interior del cuello existía en fr. ant. gorju o gorgu 'gargantón'. Para la zona de más abajo

${ }^{25}$ Oliva y Buxton, op. cit., pág. 181. Los ejemplos catalanes se han tomado también de Massó i Ruhí, M. Lluisa, Carlos Subirats Rüggeberg, y Phillippe Vasseux, Diccionari invers de la llengua catalana, Bellaterra, Universitat Autònoma de Barcelona y Laboratoire d'automatique documentaire et linguistique, 1985, págs. 404-405. 
del cuello existía en fr. ant. nervu 'atrevido, fuerte', epaulu 'ancho de espaldas', ossu 'huesudo' (mod. osseux), coillu 'no castrado' y jambu 'de piernas, de patas', además de corsu 'corpulento'; la palabra atestiguada corporu (ídem) parece ser un semicultismo, derivado de la raíz del caso oblicuo CORPOR- y no del nominativo/acusativo CORPUS. La extensión del significado del fr. ant. costu, de coste 'costilla', no se refiere a un animal o una persona de costillas prominentes — como el catalán costellut - sino a una madera de trepa acanalada ${ }^{26}$.

Con el paso del tiempo han dejado de existir muchos de los adjetivos en $-u$. De hecho, todos los adjetivos que aparecen en el párrafo anterior son desusados hoy.

De igual manera, Huguet hace notar que muchos adjetivos en $-u$ que aparecen en obras del siglo XVI han desaparecido sin dejar rastro. Muchos de ellos parecen haber sido aumentativos ${ }^{27}$. Sin embargo hoy:

[n]ous ne pouvons plus parler des corbeaux becus, du lierre brassu, des hommes corporus... du coq crestu, des chevaux crinus, des animaux dentus, d'un homme espaulu... d'un dragon griffu, d'un oison jambu, d'un corps mal ossu...

Huguet señala que en los poemas franceses del siglo XVI todavía se podía describir un lago con la palabra jonchu 'juncoso', y las piedras con veinu 'venoso'. Huguet nota que en la poesía pastoral de aquella época se encontraba también lainu o lanu 'lanoso', houpu 'empenachado' y houssu 'espeso, tupido' (los dos últimos hacen referencia al vellocino). Huguet define el último término con la palabra touffu 'empenachado', de touffe, ilustrando de modo indirecto que siguen existiendo algunos adjetivos en $-u$.

¿Por qué decayó en francés el uso de los adjetivos en $-u-\mathrm{y}$ también en -eux - a lo largo de los años? Una posible respuesta es la siguiente. A lo largo de los siglos un fuerte desgaste fonético ha disminuido las posibilidades morfológicas para los aumentativos y los diminutivos en general. Dada la síncopa de las sílabas postónicas y la reducción a cero de las consonantes mediales, por ejemplo en queue 'cola' <CAUDA, algunos de estos adjetivos no tuvieron éxito posiblemente porque los hablantes de francés fueran reacios a la formación de una monstruosidad como *queue-u o *cou-u 'rabudo', que hubieran contenido unas vocales en hiato. Sin embargo, en fran-

${ }^{26}$ Douglas C. Walker, Dictionnaire inverse de l'ancien français, Ottawa, Éditions de l'Université d'Ottawa, 1982, passim.

${ }^{27}$ Edmond Huguet, Mots disparus ou vieillis depuis le XVI siècle, 2.a ed. (Etudes de philologie et d'histoire, 2), Geneva, Droz, 1967, págs. 167-169. 
cés antiguo existía coé 'con rabo', que no tenía valor aumentativo, pero sí tenía hiato. El portugués, que también sufrió una erosión fonética, insertó una [z] antihiática en los aumentativos mãozudo y cuzudo de mão 'mano', cu 'trasero', y posiblemente también en pezudo de pé 'pie' ${ }^{28}$. De igual manera, el catalán parece tener una $[\mathrm{g}]$ antihiática en algunos adjetivos de esta clase.

Sin embargo, una explicación basada simplemente en la fonotáctica no es suficiente para dar cuenta de la desaparición de los adjetivos del francés antiguo como crestu, crenu y ossu que todavía resultarían bien formados hoy. Parte de la respuesta puede estar en que en francés los sufijos aumentativos y diminutivos son menos productivos que en las lenguas romances que se encuentran más al sur, o precisamente en latín; ya en francés antiguo su productividad era limitada y desde la época en cuestión ha disminuido más. Uno se puede preguntar por qué en francés se siguen manteniendo algunos adjetivos de esta clase. Los adjetivos en $-u$ no son de momento una reliquia morfológica, pero parece que van camino de serlo.

En una observación de talante particularmente francés, Huguet atribuye la desaparición de muchos de los adjetivos en - $u$ al «désir de clarté» que tienen los hablantes nativos: las perífrasis como au long bec u aux longs bras han reemplazado becu, brassu ${ }^{29}$. Sin embargo, las mismas perífrasis que emplea Huguet sugieren que el declive de los adjetivos en $-u$ tiene que ver más con una disminución de la tolerancia francesa hacia los diminutivos y los aumentativos en general. Esta interpretación es apoyada por la observación de Huguet (143) de que en las palabras del siglo XVI como fendasse 'fisura grande' y villasse 'ciudad grande', el sufijo -asse<-ACEA todavía añadía un matiz aumentativo en vez del matiz peyorativo que tiene hoy. Finalmente, como nota Stefanini, en Francia los gramáticos preceptivos han mantenido desde el siglo XVII una campaña en contra de las «formazioni alternative», sobre todo las que llevan una carga emotiva ${ }^{30}$. En gran parte, esta campaña ya se ha ganado.

\section{LOS ADJETIVOS ITALIANOS EN -UTO QUE SE REFIEREN A PARTES DEL CUERPO}

Además de un adjetivo en -uto, occhiuto 'con ojos, con muchos ojos, vigilante en exceso', en italiano existe un derivado, occhialuto 'con anteojos'

\footnotetext{
${ }^{28}$ Como ha notado un lector, [z] puede haber sido parte de pezudo originalmente (cf. PE$D E M, P E D E \bar{S}$ ), aunque la [d] intervocálica normalmente desapareció en portugués (Williams, $o p$. cit., pág. 68)]; a continuación [z] posiblemente se extendiera a otras partes del cuerpo.

${ }^{29}$ Huguet, op. cit., pág. 169.

${ }^{30}$ Ruggero Stefanini, Alterazione e Derivazione nominale intorno alle "Parti del corpo» in italiano, Archivio Glottologico Italiano LXVII, 1-2, 1982, págs. 136-165.
} 
que aparece en Battaglia (cf. en esp. el peyorativo anteojudo). Algunos adjetivos de esta clase son raros como boccuto 'bocudo' y linguto 'de lenguaje sarcástico', algunos son dialectales como testuto 'cabezudo' y otros, arcaicos como spalluto 'ancho de hombros', crestuto 'crestado', carnuto 'carnoso' y nervuto 'atrevido, fuerte' ${ }^{31}$.

Si testuto de TESTA 'olla, cráneo' suena una palabra del norte (cf. fr. têtu), el gentilicio Caputo 'cabezudo', de CAPUT, viene de los dialectos del sur donde la palabra para 'cabeza' en todos sus sentidos todavía es capo en vez de testa, y sugiere que Caputo tuvo su origen como apodo vernáculo. En el norte, donde la lenificación todavía existe, el antiguo milanés tenía malastrudho ( $d h$ es la grafía que representa $[\partial]$ ), aparentemente derivado de $\operatorname{MAL}(E)+\operatorname{ASTR}(\mathrm{U})+$-UTU con el significado 'de mal agüero', es decir 'desgraciado' o 'desdichado'. Esta palabra aparece en los versos de Bonvesin de la Riva (ca. 1250-ca. 1315).

El italiano estándar también tiene otros adjetivos en -uto que no se mencionan en la tabla que aparece abajo. Con referencia al pelo existen ricciuto y riccioluto 'rizado', de riccio(lo) 'rizo'. En cuanto a los tipos de cuerpo, existe el adjetivo cicciuto 'carnoso', de ciccia, con el que se puede comparar el término ciccione 'gordo' y el apodo de una representante parlamentaria conocida como La Cicciolina.

No todos los términos aquí recogidos sirven como puros aumentativos. Cuando se refiere al terreno, por ejemplo, el it. dorsuto significa 'montuoso, montañoso', sin duda por la imagen de unos seres humanos agachados con la columna vertebral expuesta, pero nunca quiere decir 'espaldudo'; un término parecido en catalán, esquenut, de esquena 'espalda', significa 'agachado, encorvado'; en portugués espinhudo 'espinoso' se refiere solamente al pescado con muchas espinas. En italiano ginocchiuto no significa 'rodilludo' sino 'abultado' o 'con protuberancias', especialmente con referencia a los troncos de los árboles. La desinencia derivada de -ŪTU también se aplica a algunos componentes léxicos más en italiano, como (bi)forcuto 'bifurcado', el arcaico ch(i)er(i)cuto 'tonsurado', es decir 'hecho clérigo', el arcaico canterutu 'anguloso', cestudo 'empenachado (dícese de las plantas), $\mathrm{y}$ el arcaico y literario fronduto o fronzuto 'frondoso'.

En el italiano estándar, como ocurre generalmente en las lenguas romances, se puede nominalizar cualquier adjetivo de forma que, por ejemplo, it. l'orecchiuto puede significar 'el orejudo', de la misma manera que una famosa actriz del siglo XIX se conocía en Francia como la Goulue 'la golosa'.

\footnotetext{
${ }^{31}$ Salvatore Battaglia, ed., Grande Dizionario della Lingua Italiana, Turín, Unione tipografico -Editrice torinese, 1961-, passim. Hasta la fecha han aparecido 16 volúmenes $(A-R o b a)$.
} 
Por otra parte, en italiano un gran número de adjetivos pueden aparecer con el sufijo superlativo -issimo que incrementa su valor aumentativo. Salvo en el caso de membruto 'membrudo, fornido', barbuto 'barba larga' y algunos más como linguacciuto 'lengua viperina', panciuto 'panzudo' y, por supuesto, cornuto 'cornudo', los adjetivos en -uto pertenecen al registro formal, en contraste con el carácter popular que tienen tales adjetivos en otras lenguas romances occidentales.

\section{LA INTRUSIÓN DE LAS DESINENCIAS DERIVADAS DE -ŌSU Y -ĀTU AL OESTE DEL ADRIÁtICO}

La mayoría de los adjetivos del francés antiguo examinados arriba tienen cognados modernos en italiano o catalán. En muchos casos el sufijo ha cambiado de $-u<-\bar{T} T$ a $-e u x<-\bar{S} \mathrm{SU}$ o $-\dot{e}<-$ ÂTU. En la Francia medieval a uno que tuviera la piel fea se le podía llamar lepru 'leproso' (mod. lepreux). De un pájaro se podía decir que era crestu 'crestado/crestudo' (mod. crêté) o реnnu 'emplumado, con plumas largas' (mod. y culto penné 'pinnado [de las hojas]') ${ }^{32}$. Se podía también describir al terreno como rochu 'rocoso' (mod. rocheux), a los árboles como ramu 'ramoso' (mod. rameux) y a la tela como lanu 'lanosa' (mod. laineux) ${ }^{33}$.

Aunque el francés prefiere en algunos casos el sufijo -eux, este sufijo también se ha vuelto menos productivo desde el siglo XVI. Al igual que $-u$, la terminación -eux indica a menudo la abundancia de la base, como hace notar Huguet ${ }^{34}$ :

Une forêt était arbreuse, la grève, areneuse; le Liban, cedreux, la nuit estoileuse; une terre, fruiteuse, les forêt, fueilleuses; une rivière, isleuse; la mer, ondeuse; la vigne, pampreuse, l'Orient, perleux, l'orangier, pommeux...

Varias de las palabras que aparecen arriba pueden haber sido inventadas por los poetas, pero ninguno de estos adjetivos en -eux ha sobrevivido hasta nuestros días. Areneux 'arenoso', basado en el desaparecido areine $<(\mathrm{H})$ ARĒNA, ha sido reemplazado por sablonneux, del mismo tipo que SABULŌSo 'arenoso, guijoso' ${ }^{35}$.

\footnotetext{
${ }^{32}$ Cf. en español moderno crestudo con connotación emotiva 'arrogante, orgulloso' y aumentativa 'con cresta grande [de los pájaros]'. En catalán crestut parece ser simplemente aumentativo.

${ }^{33}$ Frédéric Godefroy, Lexique de l'ancien français, eds. J Bonnard y A. Salmon, París, Champion, 1982, passim.

${ }^{34}$ Huguet, op. cit., pág. 163.

${ }^{35}$ No es inverosímil pensar que areneux sobrevivió de (H)ARĒNŌSU, que se encuentra en Virgilio y Ovidio.
} 
Otros adjetivos derivados con $-u$ que han sobrevivido han cambiado de forma. Así, maillu 'hecho de mallas' ha cedido su puesto al moderno maillé 'tejido de mallas', marbru 'hecho de mármol' a marbré 'con aspecto de mármol' y crostu 'costroso' de croste 'costra, corteza' al moderno croûteux 'con aspecto de costra'. En el francés antiguo a un caballo se le podía llamar crenu o grenu 'con crines largos', y todavía es así en italiano crinato o crinito y en catalán crinut; en el francés moderno todavía existe crenu o grenu 'con muesca' de cran 'muesca, diente de una rueda', pero cf. también el moderno crené 'con muesca'.

En otras lenguas romances los adjetivos con terminaciones de -ŪTU coexisten con los adjetivos con desinencias derivadas de -ĀTU en formas secundarias como el port. campanado y los it. biforcato y capellato, aunque para el último la variante en -uto, capelluto se da mucho más frecuentemente. De la misma manera, en catalán camat está en competencia con cam [arr]ut ${ }^{36}$. En vez de algo como *corpudo, el portugués tiene encorpado 'corpulento', y en algunas variedades del español se da más a menudo lengado 'hablador' que lengudo.

En español también parece que en cierta medida está disminuyendo la frecuencia de los adjetivos en -udo. Por ejemplo, el diccionario de la Real Academia de 1924 define calmudo como calmoso, cerdudo como cerdoso, hojudo como hojoso y velludo como velloso. Ese diccionario también define lengudo como lenguaraz ${ }^{37}$. El diccionario de la Academia de 1992 va más lejos, y remite el aumentativo caldudo a caldoso, cosquilludo a cosquilloso, escamudo a escamoso, y también el favorable talentudo a talentoso ${ }^{38}$. De esta manera tienden a predominar las formas secundarias en -oso para algunos items léxicos en español; el italiano tenía ventroso 'ventrudo', pero parece que nunca existió *ventruto, y el italiano todavía tiene fegatoso 'colérico, irritable', de fegato 'hígado'. En un caso de sustitución del sufijo, el informante italiano se acuerda de haber escuchado (de un niño pequeño) la palabra riccioloso 'rizado' en vez del estándar riccioluto.

Para estos términos léxicos las formas secundarias del adjetivo en -oso<-ōsu tienen tendencia a reemplazar algunos de los adjetivos en - $u d o$. De hecho, varios de los adjetivos catalanes en -ut como rabiut, golut, tienen las for-

\footnotetext{
36 Sin embargo, según Oliva y Buxton (pág. 170), la forma secundaria camat tiene que ser precedida por un adverbio, por ejemplo ben camat 'con piernas bien proporcionadas' o mal camat 'con piernas mal proporcionadas', y por lo tanto no se puede considerar un morfema libre, ya que está ligado al adverbio precedente.

37 Diccionario de la lengua española, Madrid, Real Academia Española, 1924.

38 Diccionario de la Real Academia Española, 1992. Esta obra también ofrece las palabras en -oso hebroso, hilachoso, mechoso, vedijoso como alternativas a los términos hebrudo, hilachudo, mechudo y vedijudo en -udo.
} 
mas paralelas en -oso, rabioso y goloso, en español. Dado que existen las formas latinas RABIŌSU y GOLŌSU, estos términos quizá representen un renacimiento más que una supervivencia.

A veces, sin duda hay que considerar que el desarrollo de los adjetivos con terminaciones de -ŪTU fue posterior al desarrollo de sus congéneres en -ĀTU. Así, el verbo fr. de la $1^{\text {a }}$ conjugación ficher 'clavar, hincar' fue rehecho sobre verbo de la 3 . $^{\text {a }}$ conjugación FīGERE 'fijar, ligar'. Con el paso del tiempo su participio pasado regular fiché se convirtió en sustituto más o menos cortés de foutu, en el sentido de 'malhecho, estropeado'. El mod. fichu tal vez sea un ingenioso cruce hecho por los hablantes, en el que se cambió la terminación del participio pasado por la $-u$ del participio pasado tabú. Mientras tanto, fiché se sigue utilizando con función verbal ${ }^{39}$.

\section{AdJetivos aUmentativos QUE SE REFIEREN A LAS PARTES DEL CUERPO EN RUMANO}

Consideremos ahora el rumano de los Balcanes ${ }^{40}$. Como es de esperar, el rumano es parecido a sus parientes occidentales en que tiene una serie de adjetivos que se refieren a partes del cuerpo y que tienen fuerza aumentativa. Sin embargo, solamente dos de ellos terminan en -ut tónico - cornut 'cornudo', de CORNŪTU y limbut 'hablador', de *LINGUUTU- ya que una tercera palabra heredada, CĀNŪTU 'cano', ha dado cărunt ${ }^{41}$. Todos los demás adjetivos aumentativos que se refieren a las partes del cuerpo terminan en -os tónico. Los que se refieren a partes por encima del cuello incluyen fruntos 'frentón', ochios 'ojos saltones', năsos 'narizón', y fălcos 'mandíbula saliente' (cf. normalmente pl. LC FAUCĒS 'garaganta, gola').

En rumano, el popular minto 'inteligente', de minte 'mente, seso', de MENTE 'mente, entendimiento', se parece al español sesudo, semántica si no etimológicamente. Con referencia a las partes del cuerpo por debajo del cuello, existen umeros 'de espalda ancha' de $u$ măr, spătos 'espalda ancha' de spate, pieptos 'pechugón' de piept, junto con ţăţoasă 'de pecho abultado' de tâță. A uno que tiene pelo aquí y allí se le puede llamar bărbos, mustăcios o păros 'pelo escaso', o perciunos 'rizo largo', de perciuni, este

\footnotetext{
${ }^{39}$ Wilhelm Meyer-Lübke, Grammaire des langues romanes, vol. II, Ginebra, Slatkine Reprints, 1974, pág. 566

${ }^{40} \mathrm{La}$ mayoría de los términos rumanos considerados aquí son del Dicţionar invers, Bucharest, Editura academiei, 1957.

${ }^{41}$ Por otra parte, MINŪTU 'aminorado, disminuido', quizá en un cruce con MINIMU 'mínimo' y después de la disimilación de la segunda consonante nasal, ha producido el adjetivo mărunt 'pequeño'.
} 
último con connotación antisemita. A un rumano obeso se le puede describir con la palabra burtos 'barrigón', de burtă, păntecos 'panzudo', de păntec, o con osănzos 'fofo', de osănză. Una persona con la columna vertebral malformada se llama ghebos 'jorobado', de gheb 'joroba', cf. it. gobbo, gibboso.

Como en otros casos, muchos adjetivos aumentativos en rumano se refieren a rasgos característicos, a menudo a rasgos poco halagüeños. Otra vez, todos los que son derivados de sustantivos que denotan partes del cuerpo terminan en -os: căpos 'cabezón' significa 'sabelotodo' o 'terco', limbos 'lengua larga' puede significar 'hablador, charlatán' y coios, de coi 'testículos', significa 'agresivo' o 'inteligente'. Un término favorable es inimos, literalmente 'corazón grande', de inimă 'corazón'<ANIMA 'alma', cuyo adjetivo significa 'generoso, servicial'.

Como siempre, los adjetivos que se refieren a partes del cuerpo de los animales tienen connotación peyorativa cuando se aplican a los humanos: en rumano colt 'colmillo' ( $\mathrm{pl}$. colţi) da colţos 'pendenciero, abusador' (también colţuros 'tosco, rudo), y bot 'hocico' da botos 'bocazas'. Compárese con esto el cat. barrut 'glotón, goloso; descarado', de barra 'mandíbula (normalmente de animales)', y el esp. picudo, que significa 'nariz puntiaguda' con referente humano; de la misma manera esp. frontudo, que se usa para los animales, sería peyorativo si se aplicara a los humanos.

En rumano, como en los otros idiomas romances, la terminación $-a t<\bar{A} T \mathrm{~T}$ también se puede usar con fuerza aumentativa, por ejemplo en urechiat 'orejón' de ureche, o en el más común buzat 'bocazas', además de buzos 'idem', los dos de buză. Finalmente, el rumano no representa la idea de 'cornudo' con cornut sino con incornorat, con desinencia derivada del siempre popular $-\overline{\mathrm{ATT}}{ }^{42}$.

\section{EsPeCIALIZACIÓN DE FUNCIONES EN EL ESTE Y EL OESTE}

Antes de llegar a una conclusión definitiva en cuanto al éxito que ha tenido el sufijo -ŪTU para los adjetivos y los participios pasados en toda Romania, hay que examinar los reflejos de los 78 verbos latinos que en todas las lenguas romances tienen o han tenido participios pasados con desinencias derivadas de -ŨTU. Si se incluyen los reflejos de los ítems co-

\footnotetext{
${ }^{42}$ Como ha notado un lector, el infinitivo incornora significa 'cornear, tirar, topetar'; como el italiano incorniciare 'enmarcar' basado en cornice, el verbo rumano puede ser usado para connotar el estado de ser cornudo.
} 
mo BATTŪTU, FUTŪTU y VOLŪTU que ya existían en LC y las innovaciones que se suponen del latín tardío como *HABUTU 'tenía' y * POTUTU 'haber podido', y si se excluyen los verbos con prefijo y las formas que claramente son cultismos, se llega al siguiente recuento en la Romania:

\begin{tabular}{lcrrrrrc}
\hline & rumano & italiano & francés & catalán & $\begin{array}{c}\text { español y } \\
\text { portugués }\end{array}$ \\
\hline Participios pasados en -uto/-udo/-ut/-u....... & 33 & 36 & 56 & 44 & 0 \\
Menos: formas arcaicas y competidoras.... & 0 & 7 & 10 & 5 & 0 \\
\hline TOTAL ....................................................... & 33 & 29 & 46 & 39 & 0 \\
\hline
\end{tabular}

El español y el portugués no tienen ni un solo participio pasado con un marcador de clase verbal derivado de -ŪTU ${ }^{43}$. El catalán tiene el segundo total de participios pasados más alto, sobrepasado solamente por el francés. Más hacia el este, el rumano y el italiano poseen cantidades respetables.

Como se expone en el Apéndice 1, un estudio de los adjetivos en -uto que se refieren a partes del cuerpo revela que éstos se dan más a menudo en catalán (48 adjetivos de este tipo encontrados), una lengua que también tiene un amplio inventario de participios pasados en -uto (39 participios pasados de este tipo encontrados) ${ }^{44}$. En italiano existe un número parecido de participios pasados (36) y de adjetivos que se refieren a partes del cuerpo (31) con terminaciones derivadas de -ŪTU, a pesar de las restricciones de registro que muestran estos adjetivos. En francés, donde hày más ejemplos de participios pasados en $[\mathrm{y}]<-\mathrm{U} T \mathrm{TU}$ que en cualquier otra lengua examinada (46 ejemplos), el número de adjetivos con esta terminación que se refieren a partes del cuerpo (actualmente 26) ha disminuido a lo largo de los siglos.

En los extremos de la Romania, esta desinencia se ha especializado con una de las dos funciones que tenía en unos cuantos participios pasados y adjetivos en LC. Aunque el español (con 42) y el portugués (con 35) tienen un gran número de adjetivos de este tipo, ninguna de estas lenguas tiene ni una sola forma verbal en - $u d o$ que se use como participio pasado. Aquí esta desinencia se ha visto limitada solamente a una función adjetival.

${ }^{43} \mathrm{Si}$ se hubiera incluido los participios pasados del español antiguo, el español por lo menos hubiera terminado con un saldo negativo.

${ }^{44}$ Los datos en el Apéndice 1 se han dividido en 5 grupos usando un criterio puramente intuitivo: a) partes del cuerpo humano por arriba del cuello; b) partes del cuerpo humano por debajo del cuello; $c$ ) partes del cuerpo de los animales; $d$ ) la pilosidad en los seres humanos; e) tipos de cuerpo humano. 
Por otra parte, a pesar de la existencia en rumano de muchos participios pasados en -ut (33), la terminación -os<-ōsu ha triunfado como desinencia para los adjetivos aumentativos derivados de las partes del cuerpo humano. En esta lengua, con unas pocas excepciones, algunas apenas reconocibles, la desinencia -ut se ha visto limitada a una función de participio pasado.

Una lengua romance dada puede tener adjetivos que se refieran a partes del cuerpo en -ŪTU, participios pasados con terminaciones de -ŪTU, o las dos cosas, en una distribución que divide Romania en tres partes. En el núcleo del romance desde París hasta Barcelona y hasta Florencia, tanto los participios pasados como los adjetivos que se refieren a partes del cuerpo con terminaciones de -ŪTU siguen floreciendo, aunque en francés menos ahora que en el pasado. El español, más occidental, y el portugués utilizan -udo solamente como terminación adjetival. El rumano, más oriental, utiliza - ut casi exclusivamente como terminación de participio pasado.

\section{ANÁlisis DE LOS DATOS RECOGIDOS EN El APÉNDICE 1}

Para ser incluido en la tabla sin paréntesis ni punto de interrogación, un término en italiano, francés, catalán, español o portugués modernos tiene que darse en un informante hablante nativo y tiene que aparecer en algún libro de consulta.

Durante la recogida de los datos, algunos informantes dieron evidencia indirecta de que el sufijo -uto, etc., sigue siendo productivo en varios vernáculos del romance. Un hablante de italiano ofreció las palabras frontuto 'frontudo', mentuto 'mentón grande' y culuto 'culón', pero ninguna de ellas aparece en Battaglia ni en Zingarelli; tampoco aparecen cazzuto 'pene grande' o polpacciuto 'agradablemente rollizo' proporcionados por otro hablante ${ }^{45}$. Un hablante catalán nunca había oído collut 'cuello largo' ni genollut 'rodilludo', lo cual sugiere que pueden ser palabras fantasma en MeyerLübke; el mismo hablante utilizó plomut 'plumoso', pero esta palabra tampoco se encuentra. Un hablante francés sugirió onglu 'uñoso', parecido al it. unghiuto, pero la palabra francesa no aparece en ningún libro de consulta, como tampoco lo hace el antiguo fr. bouchu 'bocudo' de Meyer-Lübke. Por otra parte, un hablante de español propuso pernudo, pero otro hablante negó la existencia de esta palabra; otro informante sugirió ojudo, pero otro dijo que tal palabra no existe. Según un hablante mexicano, trompudo significa 'labio grueso, bocudo', pero según un hablante peninsular significa

\footnotetext{
${ }^{45}$ Nicola Zingarelli, Vocabulario della lingua italiana, 10. ed., Bologna, Zanichelli, 1970.
} 
'con trompa, como los elefantes', y la palabra no aparece en el diccionario de la Real Academia de 1992.

A pesar del desacuerdo existente, el sufijo aumentativo de -ŪTU se puede aplicar no solamente a las palabras heredadas del latín que denotan partes del cuerpo, sino también a algunas palabras prestadas del germánico, como el it. gotuto 'mofletudo', fr. mafflu 'idem.' y lippu 'labio grueso'. De cachaza 'ociosidad, lentitud', derivado del turco, viene el esp. cachazudo.

Entre las lenguas estudiadas para los adjetivos con desinencias de -ŪTU, solamente el portugués tiene una palabra que significa 'pies grandes'; solamente el español y el italiano tienen términos con el significado de 'pantorrilludo'; solamente el español puede tener una palabra que se refiere a los genitales de gran tamaño; solamente el catalán tiene dos términos que significan 'cuello grueso'; el francés antiguo tenía dos palabras con el significado de 'gargantón'. Sólo en portugués existe cambudo 'nariz respingona', palabra desconocida para el informante portugués que puede ser otra palabra fantasma, pero que sin embargo se parece al fr. camus 'idem'. La existencia de algunos adjetivos muy difundidos como it. manuto, cat. manut, port. mãozudo 'manota' — que parecen no tener cognados en francés ni en español- hace suponer que estos términos o sobrevivieron en las tres lenguas, o siguieron un camino paralelo a la morfología aumentativa.

Quiero dar las gracias a los hablantes nativos que han contribuido a este estudio: para el rumano, Emil Vrabie; para el italiano, Ruggero Stefanini; para el francés, Edwige Gamache; para el catalán, Joan Sempere; para el español, Antonio Cortijo y Marta Mendoza; para el portugués, Milton Azevedo. La traducción del inglés al español fue hecha por Regina Morin. 
APÉNDICE 1. Adjetivos en -UTO, etc., que se refieren a las partes del cuerpo, de Italia hasta Portugal

\begin{tabular}{|c|c|c|c|c|c|}
\hline glosa & italiano & francés & catalán & español & portugués \\
\hline \multicolumn{6}{|l|}{$a$. } \\
\hline FRENTE & frontuto*? & - & - & ceñudo+ + & - \\
\hline OJOS & occhiuto* & - & ullut* & ojudo* & olhudo* \\
\hline NARIZ & nasuto* & - & nassut* & narigudo* & narigudo* \\
\hline OREJAS & orecchiuto* & (oreillu*) & orellut* & orejudo* & orelhudo* \\
\hline MEJILLAS & gotuto* & $\begin{array}{l}\text { joufflu*, } \\
\text { mafflu* }\end{array}$ & galtut & cachetudo & - \\
\hline BOCA & boccuto* raro & (bouchu*)? & bocut & bocudo* & bocudo* \\
\hline LABIOS & labbruto* & $\begin{array}{l}\text { (lavru*) } \\
(\text { levru*) } \\
\text { lippu* }\end{array}$ & morrut* & $\begin{array}{l}\text { bezudo*, } \\
\text { trompudo*? }\end{array}$ & beiçudo* \\
\hline DIENTES & dentuto* & (dentu*) & dentut* & dentudo* & dentudo* \\
\hline LENGUA & $\begin{array}{l}\text { (linguto+) } \\
\text { linguarudo+ }\end{array}$ & - & llengut+ & lengudo+ raro & lingudo*, \\
\hline GARGANTA & - & $\begin{array}{l}\left(\text { gorju}^{*}\right) \\
\left(\text { gorgu }^{*}\right)\end{array}$ & górjut* & - & - \\
\hline $\begin{array}{l}\text { BARBILLA/ } \\
\text { QUIJADA }\end{array}$ & $\begin{array}{l}\text { bazzuto*, } \\
\text { mentuto*? }\end{array}$ & - & queixut*+ & - & queixudo*+ \\
\hline CUELLO & - & 一 & collut*? & - & pescoçudo* \\
\hline NUCA & - & - & clatellut & - & - \\
\hline CABEZA & testudo* dial. & $\begin{array}{l}\text { têtu+ } \\
(\text { testu*+) }\end{array}$ & cabut+ & $($ cabezudo*+) & cabeçudo+ \\
\hline SESO & (cervelluto + ) & - & - & sesudo+ & sisudo+ \\
\hline \multicolumn{6}{|l|}{$b$. } \\
\hline HOMBROS & (spalluto*) & (espaulu*) & espatllut* & hombrudo* & ombrudo* \\
\hline ESPALDA & dorsuto $\div$ & - & esquenut $\div$ & - & - \\
\hline BRAZOS & - & - & braonut* & - & braçudo* \\
\hline MANOS & manuto* & - & manut* & - & mãozudo* \\
\hline UÑAS & unghiuto* raro & onglu*? & - & - & - \\
\hline PEZONES & popputa* & mamelue* & mameluda* & tetuda* & tetuda* \\
\hline PECHO & - & - & mamelut* & pechudo*? & peitudo* \\
\hline COSTILLAS & - & $(\operatorname{costu} \div)$ & costellut* & - & - \\
\hline VIENTRE & - & ventru* & ventrut* & $\begin{array}{l}\text { ventrudo*, } \\
\text { barrigudo* }\end{array}$ & barrigudo* \\
\hline HÍGADO & - & - & fetjut+ & - & - \\
\hline CADERAS & - & - & ancut* & ancudo* & bundudo* \\
\hline NALGAS & naticuto+ & fessu* & - & nalgudo* & cuzudo* \\
\hline PENE & (cazzuto) & - & - & carajudo+? & - \\
\hline TESTES & - & (coillu+) & collonut+ & cojonudo+ & - \\
\hline PIERNAS & gambuto* & (jambu) & $\begin{array}{l}\text { camut* } \\
\text { camarrut* }\end{array}$ & pernudo* & pernudo*? \\
\hline RODILLAS & gionocchiuto) & - & genollut*? & - & joelhudo*? \\
\hline PANTORILLAS & polpacciuto* & - & - & pantorrilludo* & - \\
\hline PIES & - & - & - & piezudo? & pezudo \\
\hline \multicolumn{6}{|l|}{$c$. } \\
\hline CUERNO & cornuto + & cornu+ & cornut & cornudo+ & $\begin{array}{l}\text { cornudo+, } \\
\text { chifrudo }\end{array}$ \\
\hline
\end{tabular}


APÉNDICE 1. Adjetivos en -UTO, etc., que se refieren a las partes del cuerpo, de Italia hasta Portugal (Continuación)

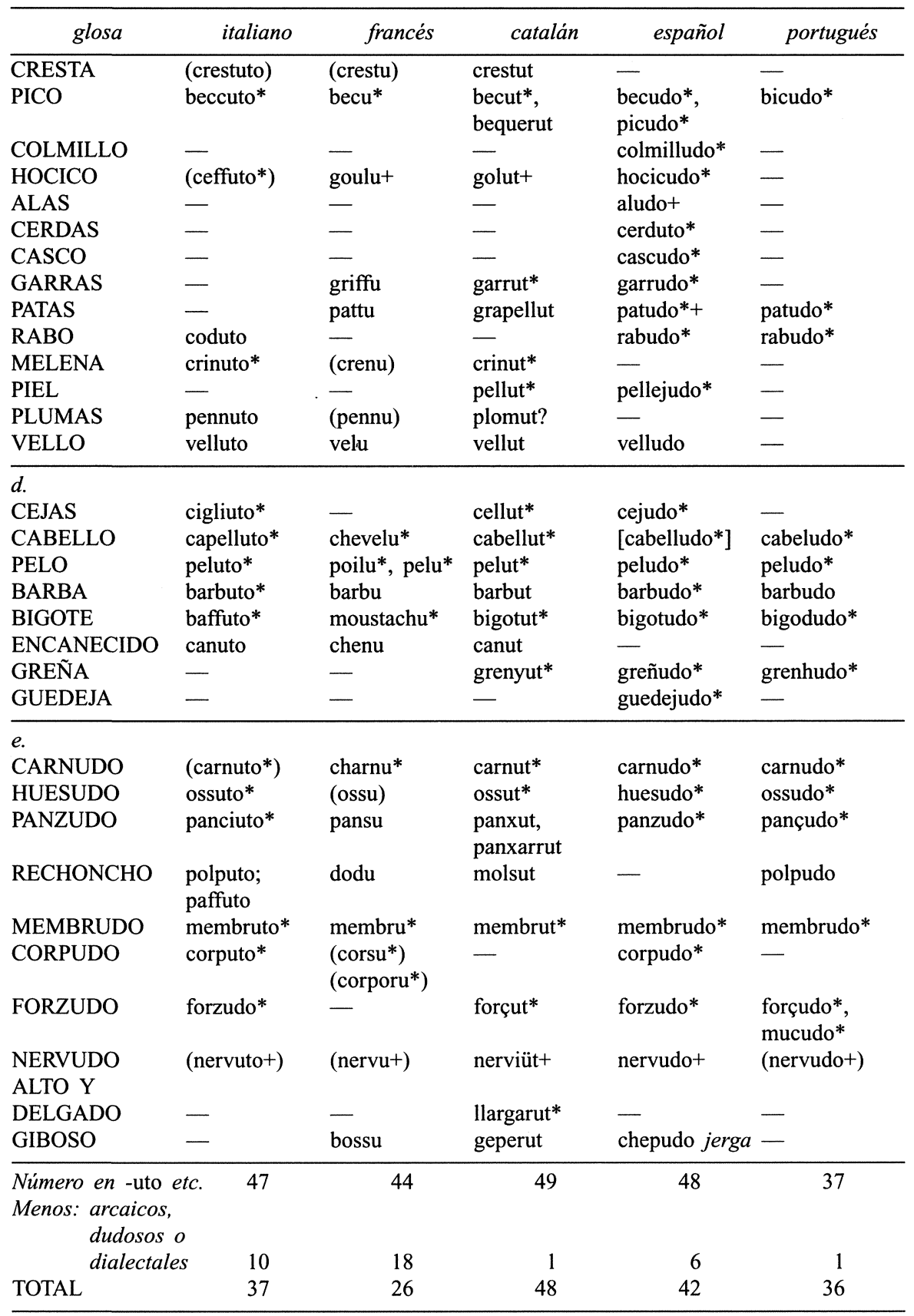

( ) = arcaico; ? = incierto; ${ }^{*}=$ aumentativo; $+=$ carácter/emociones; $\div$ más abstracto. 